\title{
A comparative ultrastructural study of pit membranes with plasmodesmata associated thickenings in four angiosperm species
}

\author{
David Rabaey • Frederic Lens • Suzy Huysmans • \\ Erik Smets • Steven Jansen
}

Received: 25 April 2008 / Accepted: 20 August 2008 / Published online: 4 September 2008

(C) Springer-Verlag 2008

\begin{abstract}
Recent micromorphological observations of angiosperm pit membranes have extended the number and range of taxa with pseudo-tori in tracheary elements. This study investigates at ultrastructural level (TEM) the development of pseudo-tori in the unrelated Malus yunnanensis, Ligustrum vulgare, Pittosporum tenuifolium, and Vaccinium myrtillus in order to determine whether these plasmodesmata associated thickenings have a similar developmental pattern across flowering plants. At early ontogenetic stages, the formation of a primary thickening was observed, resulting from swelling of the pit membrane in fibre-tracheids and vessel elements. Since plasmodesmata appear to be frequently, but not always, associated with these primary pit membrane thickenings, it remains unclear which ultrastructural characteristics control the formation of pseudo-tori. At a very late stage during xylem differentiation, a secondary thickening is deposited on the primary pit membrane thickening. Plasmodesmata are always associated with pseudo-tori at these final developmental stages. After autolysis, the secondary thickening becomes electron-dense and persistent, while the primary thickening turns transparent and partially or entirely dissolves. The developmental patterns observed in the
\end{abstract}

\footnotetext{
D. Rabaey $(\bowtie) \cdot$ F. Lens $\cdot$ S. Huysmans $\cdot$ E. Smets

Laboratory of Plant Systematics, Institute of Botany and Microbiology,

Kasteelpark Arenberg 31, P.O. Box 2437, 3001 Leuven, Belgium

e-mail: david.rabaey@bio.kuleuven.be

E. Smets

National Herbarium of the Netherlands,

Leiden University Branch,

P.O. Box 9514, 2300 RA Leiden, The Netherlands

S. Jansen

Jodrell Laboratory, Royal Botanic Gardens, Kew,

Richmond, Surrey TW9 3DS, UK
}

species studied are similar and agree with former ontogenetic studies in Rosaceae, suggesting that pseudo-tori might be homologous features across angiosperms.

Keywords Ontogeny · Pseudo-torus · Tracheary element . Pit membrane $\cdot$ Bordered pit $\cdot$ Wood ultrastructure

\section{Introduction}

The process of bordered pit formation in secondary xylem of plants has received attention by various authors (Schmid 1965; Schmid and Machado 1968; Chaffey et al. 1997). Several studies illustrated that bordered pit membranes are outlined in the primary wall of cambial derivatives by cortical microtubules (Uehara and Hogetsu 1993; Chaffey et al. 1997, 1999, 2000; Funada et al. 1997, 2001; Oda et al. 2005). These microtubules determine the cell wall pattern by guiding the movement of the cellulose synthesizing complexes (Gunning and Hardham 1982). While some bordered pits develop from primary pit fields, which are areas of the primary wall with many plasmodesmata, it is illustrated that plasmodesmata are not essential for pit development (Kerr and Bailey 1939; Carr 1976; Juniper 1977; Barnett and Harris 1975; Barnett 1981, 1982). Interestingly, the distribution of plasmodesmata in developing pits seems to be associated with particular cell types: vessel element and tracheid pits usually develop in areas without plasmodesmata, while plasmodesmata are strongly associated with the development of fibre and xylem parenchyma pits (Yang 1978; O’Brien 1981; Barnett 1982).

Relatively little attention has been paid to the development of pit membranes in hardwoods (Schmid and Machado 1968; Butterfield and Meylan 1982; Barnett 1987b). The formation of the pit membrane is accompanied 
by changes in the primary cell wall (Fengel 1972; Imamura and Harada 1973). Schmid and Machado (1968) noticed the absence of plasmodesmata in differentiating pit membranes of vessels, which were reported to swell prior to hydrolysis (Esau and Charvat 1978). This swelling is restricted to all future pit membranes where no secondary cell wall material is deposited (Catesson 1983; Singh 1987).

A special type of pit membrane formation has been recorded by Parameswaran and Liese $(1969,1973)$ : pad-like structures were found to be associated with fibre-tracheid pit membranes in Ribes sanguineum. The thickenings are highly variable in structure and are associated with the presence of plasmodesmata (Rabaey et al. 2006, in press; Jansen et al. 2007). A similar type of thickening was observed in several genera belonging to Eleaegnaceae, Ericaceae, Grossulariaceae, Oleaceae, Rhamnaceae and Rosaceae (Parameswaran and Liese 1981; Parameswaran and Vidal Gomes 1981; Barnett 1982, 1987a, b; Lachaud and Maurousset 1996; Rabaey et al. 2006, in press; Jansen et al. 2007). These plasmodesmata associated thickenings were called 'pseudo-tori' by Rabaey et al. (2006) due to a superficial similarity with genuine tori of gymnosperms as observed by light microscopy. Nevertheless, electron microscopic observations revealed that obvious differences between tori and pseudo-tori exist. Tori are defined as a central lens-shaped thickening of the pit membrane surrounded by a porous region of the pit membrane, called the margo (IAWA Committee 2004). Although tori are commonly known as a characteristic feature in conifer wood, they have been observed in a restricted number of angiosperm families as well (Cannabaceae, Oleaceae, Rosaceae, Thymelaeaceae, Ulmaceae) (Dute et al. 1992, 2004; Jansen et al. 2004, 2007; Rabaey et al. in press). Tori are present on all pit membranes of conifer tracheids, and in vasicentric tracheids or narrow vessel elements of angiosperms. Functionally, they are associated with round pit apertures. Pseudo-tori, on the other hand, are composed of an irregular thickening frequently composed of different cores, which do not always take a central position on the pit membrane. Other features characterising pseudo-tori are their inconsistent distribution throughout fibre-tracheids and narrow vessel, and the occurrence of an electron dense, cap-like thickening, which partially covers an electron transparent pit membrane as observed by TEM (Rabaey et al. 2006, in press; Jansen et al. 2007).

Tori have been demonstrated to develop according to two different ontogenetic pathways within angiosperms. They develop prior to secondary wall initiation and are not lignified in Celtis and Ulmus, while tori in Osmanthus and Daphne develop during secondary cell wall formation and are suggested to be lignified (Dute and Rushing 1988, 1990; Dute et al. 1990; Coleman et al. 2004). To our knowledge, the development of pseudo-tori has only been studied in two Rosaceae species, namely Sorbus aucuparia and Pyrus communis (Barnett 1987a, b). It is unknown whether pseudo-tori in other plant families follow a similar developmental pattern. We aim to address the question whether or not pseudo-tori show a similar developmental sequence across four unrelated species within angiosperms.

\section{Materials and methods}

Small, 1- to 2-year old branches (5-8 $\mathrm{mm}$ in diameter) were collected in June 2007 at the National Botanic Garden of Belgium from the following four species (accession numbers between brackets): Malus yunnanensis C.K. Schneid. (Rosaceae, Rosales; BR 1982 0511), Ligustrum vulgare L. (Oleaceae, Lamiales; BR 1955 2528), Pittosporum tenuifolium Gaertn. (Pittosporaceae, Apiales; BR 1975 0509), and Vaccinium myrtillus L. (Ericaceae, Ericales; BR 1980 0770).

Small stem segments were cut into $2 \mathrm{~mm}^{3}$ pieces and fixed overnight in $2 \%$ glutaraldehyde at $\mathrm{pH} 7.4$, buffered with $0.05 \mathrm{M}$ sodium cacodylate fixative at room temperature. After washing in $0.05 \mathrm{M}$ sodium cacodylate buffer, the specimens were postfixed in $1 \%$ buffered osmium tetroxide for $4 \mathrm{~h}$ at room temperature, washed, and dehydrated through a graded ethanol series. The ethanol was gradually replaced with LR White resin (London Resin Co, Reading, UK) over several days. The resin was polymerized at $60^{\circ} \mathrm{C}$ for $24 \mathrm{~h}$. Embedded samples were trimmed using a Leica EM specimen trimmer (Leica Microsystems, Vienna, Austria) and sectioned with an ultramicrotome (Ultracut, Reichert-Jung, Vienna, Austria). Transverse semi-thin sections of the cambial zone were cut with a glass knife and heat-fixed to glass slides, stained with $0.5 \%$ toluidine blue in $0.1 \mathrm{M}$ phosphate buffer and mounted in DPX (Agar Scientific, Stansted, UK). Transverse ultra-thin sections ( $\pm 50-90 \mathrm{~nm}$ ) were cut using a diamond knife and stained with uranyl acetate and lead citrate using a Leica EM ultrostainer (Leica Microsystems, Vienna, Austria). Observations were carried out using a Jeol JEM-1210 (Jeol Ltd, Tokyo, Japan) at $80 \mathrm{kV}$ accelerating voltage and digital images were taken using a MegaView III digital camera (Soft Imaging System, Münster, Germany) at the Royal Botanic Gardens, Kew. Some pictures were also taken with a Jeol JEM-1011 at $80 \mathrm{kV}$ accelerating voltage at the Jeol European Institute, Hertfordshire, UK.

Quantitative characters were measured using Carnoy 2.1 (Schols et al. 2002) and were based on 25 counts. Wood anatomical terminology follows the IAWA Committee (1989).

Thin sections $(10-15 \mu \mathrm{m})$ for light microscopy were prepared using a sliding microtome (Reichert, Vienna, Austria) and stained with safranin. After staining, sections were washed with distilled water, dehydrated with ethanol and treated with clearing agent Parasolve (Prosan, Merelbeke, 
Belgium). The sections were embedded in Euparal (Agar Scientific Ltd, Essex, UK). LM-observations were carried out with a Dialux 20 (Leitz, Wetzlar, Germany) fitted with an oil immersion objective. Digital images were made with a PixeLINK PL-B622CF camera.

\section{Results}

Pit membrane thickenings are present on pit membranes of the radial and tangential walls of tracheary elements in the four species studied. Pseudo-tori are most common on pit membranes between fibre-tracheids, but occasionally occur on pit membranes between a vessel element and a fibretracheid, and between a fibre-tracheid and a parenchyma cell (Figs. 1, 11). When a thickening is formed on a pit membrane of a pit pair between a parenchyma cell and a tracheary element, a pseudo-torus develops only at the side of the tracheary element, while a protective layer is formed on the parenchyma side (Fig. 11).

Various ontogenetic stages could be distinguished on single sections, with immature stages obviously more common near the cambium, although in all species a mix of immature and fully developed stages is found many cell layers away from the cambium. Sometimes different developmental stages occur in a single pit pair (Fig. 2). Our samples, in which the cambial activity has already decreased and a thin secondary cell wall is present in all cells near the cambium, allowed observations of selected developmental stages of pseudo-tori. The development of pseudo-tori follows a similar pattern in all species studied and no interspecific developmental differences could be detected. Therefore, we provide a general description for the species investigated.

\section{Formation of the primary thickening}

During the earliest stages observed, all pit membranes show swelling (Fig. 3). Some pit membranes undergo a substantial extra swelling, resulting in a primary thickening that is twice as thick as the initial pit membrane (Table 1) (Figs. 4, 5 , and 6). The average thickness of this primary wall is between $440 \mathrm{~nm}$ and $660 \mathrm{~nm}$ in V. myrtillus and L. vulgare respectively. This primary thickening shows the same electron density as the pit membranes that do not develop pseudo-tori. In most cases, this primary thickening stretches out over the entire length of the pit membrane, with its thickest part in a more or less central position, although a more eccentric position is also observed and occasionally two thickenings are observed in a single pit membrane (Figs. 6, 7, 10). The middle lamella can sometimes be clearly distinguished from the primary cell wall layers (Fig. $3,4)$. Plasmodesmata are seen in some pit membranes with a primary thickening (Figs. 5, 6 and 7), but never in pit membranes without thickenings. Whether these plasmodesmata are functional is not tested in this study. The plasmodesmata are radially oriented in the thickening (Figs. 5, 11). The maximum number of plasmodesmata observed in a primary thickening of one section is nine, with an average of five plasmodesmata per primary thickening for all species investigated.

\section{Formation of a secondary cap}

During an advanced stage of cell differentiation, a secondary, cap-like layer with a different chemical composition is formed on top of the primary thickening (Figs. 7, 8). At least some of the plasmodesmata occurring in the primary thickening seem to be continuous through the secondary thickening. In some cases, these plasmodesmata entirely cross the outer layers of the secondary thickening. The average thickness of the secondary thickening varies from $170 \mathrm{~nm}$ in V. myrtillus to $400 \mathrm{~nm}$ in P. tenuifolium (Table 1).

\section{Autolysis}

Finally, autolytic enzymes are released in the cell causing programmed cell death: the cytoplasm and the entire pit membrane, including the thickening, undergo autolysis (Srivastava and Singh 1972) (Fig. 9). This process appears to be initiated from the centre of the primary thickening (e.g. compare Figs. 9 and 10) and results in partial or complete breakdown of the primary thickening. The secondary thickening is much more resistant than the primary thickening, forming a persistent cap-like structure (Fig. 10). Remnants of plasmodesmata frequently remain visible in the secondary thickening. After autolysis, this secondary thickening turns red (LM) when stained with safranin suggesting that the cap is lignified, while the remaining parts of the pit membrane (including the primary thickening) become electron transparent in TEM sections. The secondary thickening thus becomes more electron dense than the primary thickening after autolysis, and the most electron dense layer is usually the inner layer of the secondary thickening (Fig. 10).

\section{Discussion}

Ontogeny of pseudo-tori

Pseudo-tori are found in the four species studied and the previously observed variation in number, size and position of pseudo-tori is confirmed. For instance, more than one pseudo-torus can occur on a single pit membrane, pseudo- 


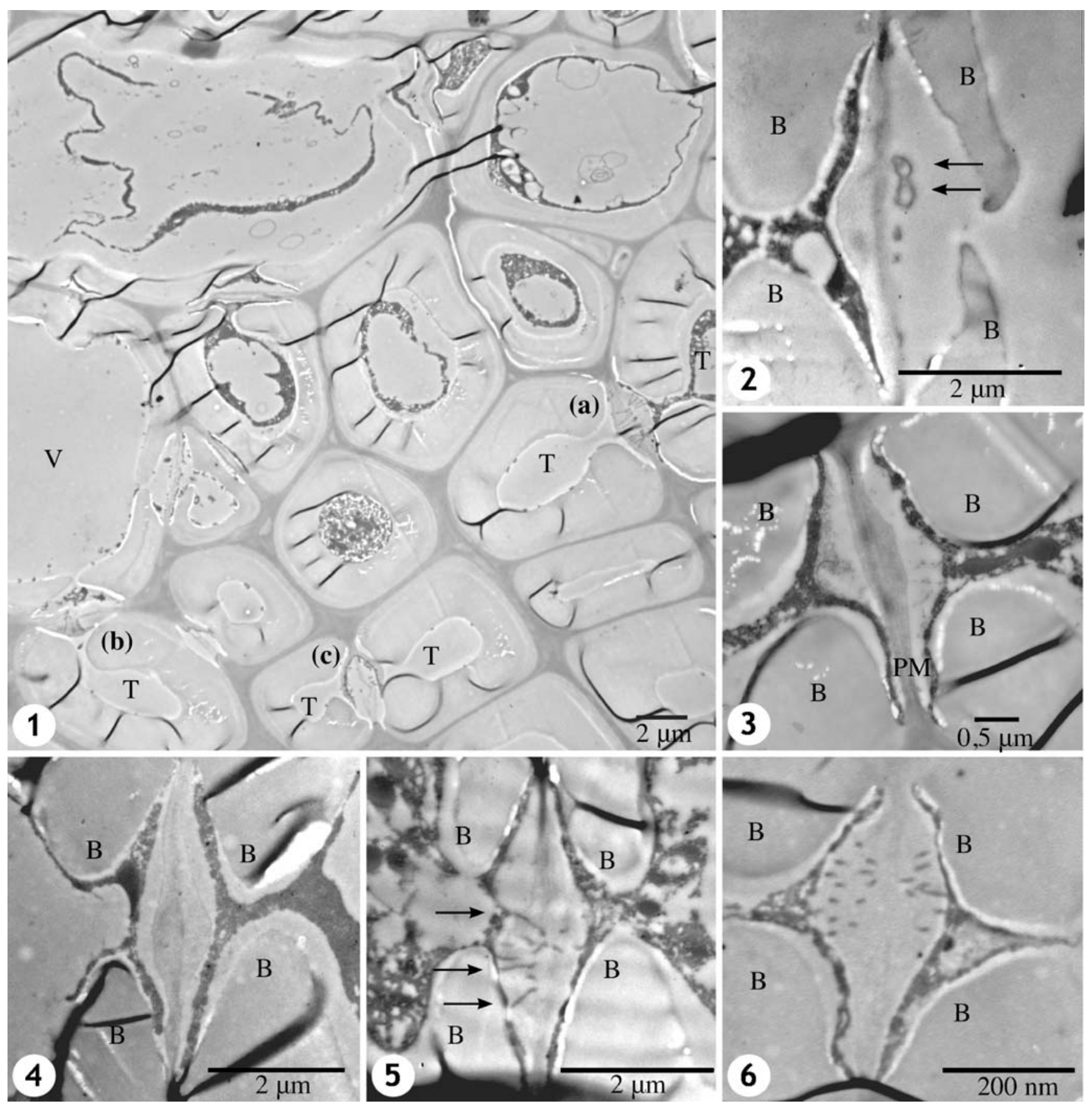

tori show a rather wide range in size and their position on the pit membrane varies from central to eccentric (Rabaey et al. 2006, in press; Jansen et al. 2007). The general developmental pattern of differentiating wood cells is well understood (Srivastava and Singh 1972). Our data indicate that the development of pit membranes with pseudo-tori follows this general pattern as described in literature (Schmid and Machado 1968; Butterfield and Meylan 1982; Catesson 1983; Singh 1987). Cambial derivative cells evolve through subsequent developmental stages into mature xylem cells. During the first stage, the cell wall consists only of primary cell wall material and the middle lamella. No secondary cell wall is formed, and the outline of future pits is not clear in ultra-thin sections. During secondary cell wall formation, primary pit membrane thickenings with or without plasmodesmata are formed on some pit membranes between narrow tracheary elements. No ultrastructural arguments are found elucidating why some pit membranes develop primary thickenings while others do not. Just before autolytic enzymes hydrolyse the protoplast, the cap-like structure is formed as the final layer of the pseudo-torus. After autolysis, the tracheary elements 
Fig. 1-6 Developmental stages of pseudo-tori ( $B=$ pit border) Fig. 1 Overview of developing xylem in Malus yunnanensis. The cambial zone is situated at the top with different ontogenetic stages visible throughout the tissue. Near the cambium, a thin secondary cell wall is formed and most cells still have cytoplasm. At the bottom, the secondary cell wall formation is completed and all cells have lost their cytoplasm. A primary thickening with plasmodesmata is present in pit pair $(a)$ on the tangential wall between two narrow adjacent tracheary elements $(T)$. Active cytoplasm can be clearly seen in the cell on the right. The autolysis of the primary thickening is initiated in pit pair (b) between a small tracheary element $(T)$ and a vessel $(V)$. Once the autolysis has completed, the electron dense secondary thickening becomes apparent in a pit membrane between two narrow tracheary elements $(T)$ in pit pair $(c)$

Fig. 2 Pit pair in Ligustrum vulgare between two narrow tracheary elements. Different stages in pseudo-torus development are present in the same pit pair. On the left, a primary thickening is being formed, while the pseudo-torus on the right side has already been hydrolysed and two parts of the persistent secondary cap are clearly visible (arrows)

Fig. 3 Formation of a primary thickening on a pit membrane (PM) between two narrow tracheary elements of Malus yunnanensis. Note the central swollen part of the pit membrane

Fig. 4 Swelling of the pit membrane and development of the primary thickening between two small tracheary elements of Malus yunnanensis. No plasmodesmata are visible at this stage of pseudo-torus development

Fig. 5 Formation of a primary thickening on a pit membrane between two narrow tracheary elements of Malus yunnanensis. Plasmodesmata are visible in the thickening as dark lines and connect the cytoplasm of both cells (arrows)

Fig. 6 Mature primary thickening with plasmodesmata between two narrow tracheary elements of Malus yunnanensis

are mature and the secondary thickening will form the most characteristic part of the pseudo-torus. Most non-cellulosic matrix compounds are thought to be removed from the pit membrane during autolysis, giving the fully developed pit membrane its transparent texture (Butterfield and Meylan 1982). The composition of the primary thickening presumably resembles that of the primary cell wall as these layers are more strongly affected by the autolytic enzymes than the secondary thickening, while the persistent nature of the secondary cap-like thickening may suggest the presence of lignin.

The developmental similarities of the four species studied agree with earlier observations in the rosaceous species Sorbus aucuparia, S. torminalis and Pyrus communis (Barnett 1987a, b; Lachaud and Maurousset 1996). Although a similar developmental pattern for all pseudo-tori may suggest their homologous nature, statements of homology need a more elaborate screening through the angiosperms to determine if all pseudo-tori also have a common evolutionary origin. Species with pseudo-tori can be found in the core eudicot order Saxifragales (Grossulariaceae), in the rosid order Rosales (Eleaegnaceae, Rhamnaceae and Rosaceae), and in the asterid orders Ericales (Ericaceae), Lamiales (Oleaeceae) and Apiales (Pittosporaceae). A common evolutionary
Table 1 Quantitative characters of pseudo-tori: (1) thickness of the primary thickening $(\mu \mathrm{m}),(2)$ thickness of secondary cap-like structure $(\mu \mathrm{m})$

\begin{tabular}{lll}
\hline Species & $(1)$ & $(2)$ \\
\hline L. vulgare & $0.30-(0.66)-1.09$ & $0.16-(0.31)-0.53$ \\
M. yunnanensis & $0.14-(0.60)-0.92$ & $0.03-(0.27)-0.48$ \\
P. tenuifolium & $0.28-(0.50)-0.78$ & $0.15-(0.40)-0.65$ \\
V. myrtillus & $0.21-(0.44)-0.67$ & $0.09-(0.17)-0.27$ \\
\hline
\end{tabular}

All data are based on 25 measurements; minimum and maximum values are given with mean values between parentheses

ancestor could be found at the base of the core eudicots, but this is highly speculative due to the restricted number of species with pseudo-tori found so far.

In $P$. communis and $S$. aucuparia, the formation of the primary thickening starts during cell enlargement and is completed before the secondary cell wall formation starts. Since our samples lack the earliest stages in cell development, the exact timing of the formation of the primary thickening cannot be determined unambiguously from our study. The main difference between our observations and those by Barnett (1987a, b) and Lachaud and Maurousset (1996) is that plasmodesmata do not always seem to be associated with the pit membrane during the development of the pseudo-torus. However, plasmodesmata are found in all pseudo-tori during later developmental stages when the primary thickening is complete and the secondary thickening is being deposited. This supports the idea that plasmodesmata in pseudo-tori show a secondary de novo nature as suggested by Barnett (1987b).

Our observations of the formation of the secondary caplike thickening and the effect of the autolytic enzymes on pseudo-tori are similar to previous ontogenetic studies of pseudo-tori (Barnett 1987a, b; Lachaud and Maurousset 1996). The thickenings observed in fibre tracheids of Ribes sanguineum, however, show a different development (Parameswaran and Liese 1973). The thickenings reported in this species consist of a primary layer associated with plasmodesmata, while a cap-like secondary thickening is absent. After autolysis, the integral structure is reported to dissolve almost completely, suggesting a cellulosic composition without high quantities of lignin. Although further research is required to investigate these pad-like thickenings in Ribes, current evidence suggests that they differ significantly from pseudo-tori.

Plasmodesmata in pit membranes and function of pseudo-tori

Our observations suggest that plasmodesmata are not essential for the development of the primary pit membrane thickening, but that they are consistently associated with 

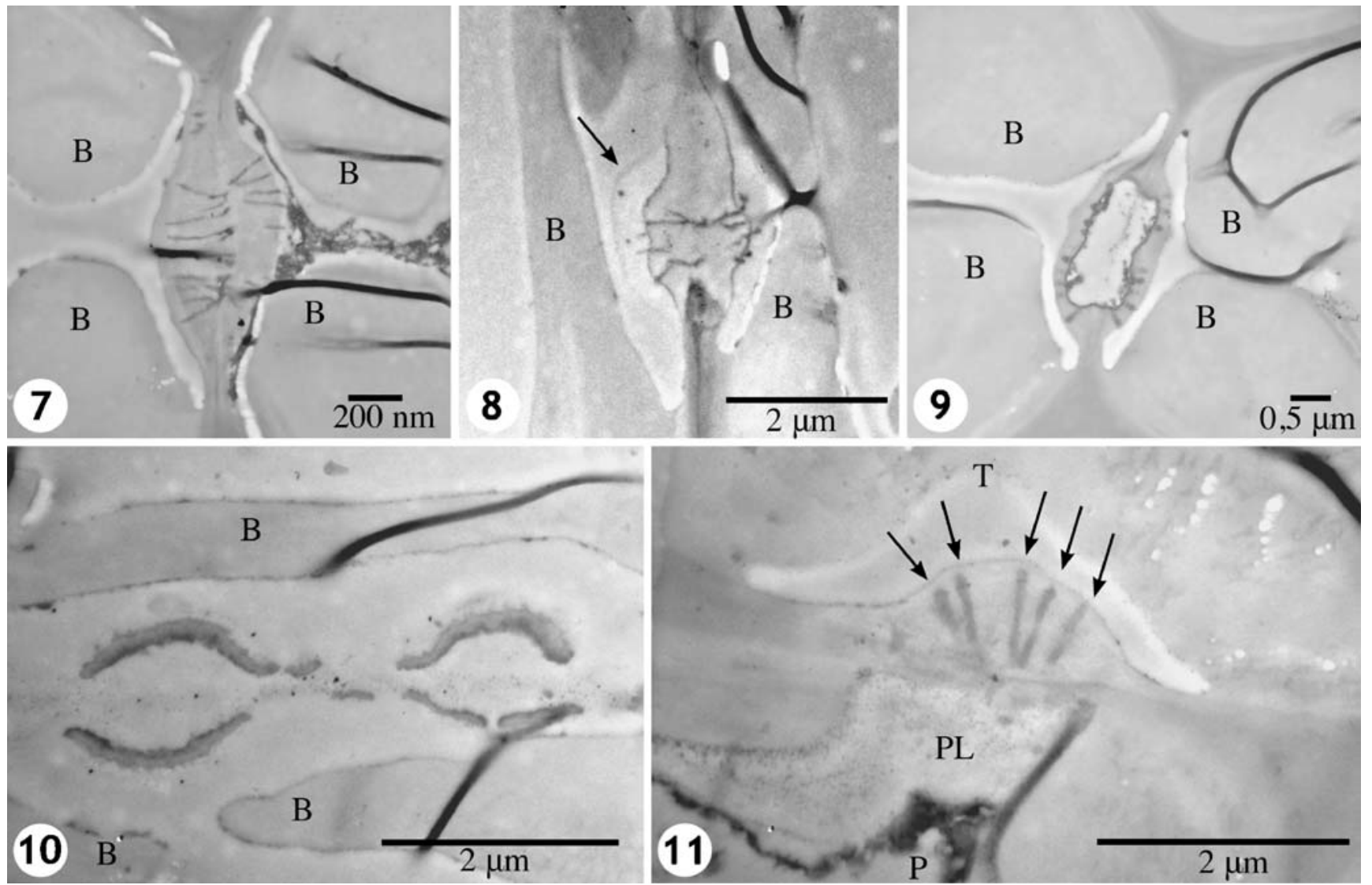

Fig. 7-11 Developmental stages of pseudo-tori ( $B=$ pit border) Fig. 7 Detail of pit pair $(c)$ from Fig. 1 (Malus yunnanensis). The primary thickening is complete on both sides and the secondary thickening has developed on the left side of the pseudo-torus. On the right side, the plasmodesmata are still in contact with the protoplast and the secondary cap has not yet been formed

Fig. 8 Pit pair with pseudo-torus just before autolysis in Pittosporum tenuifolium. The secondary cap (arrow) is present on top of the primary thickening

Fig. 9 Breakdown of the primary thickening in Malus yunnanensis

this primary thickening during later ontogenetic stages. Therefore, the most important structural difference between pit membranes with and without pseudo-tori is the presence of plasmodesmata. No experimental data on the actual functionality of plasmodesmata in pseudo-tori is available. Functional plasmodesmata play an important role in controlling the plant's developmental and physiological processes (Ehlers and Kollmann 2001). The occurrence of plasmodesmata in the primary thickening, assuming that they are functional, allows for the connection of the protoplast of two adjacent cells, while formation of the secondary cap blocks most of these connections. A similar occlusion was suggested by Barnett (1987b) and a protective role in cell differentiation was hypothesized. In this way, pseudo-tori would prevent leaking of autolytic enzymes into an adjacent cell. Consequently, tracheary elements with pseudo-tori could delay programmed cell during autolysis starting in the centre of the thickening. The secondary cap becomes more electron dense

Fig. 10 Two mature pseudo-tori (arrows) associated with a single pit membrane of Vaccinium myrtillus. Note the electron dense layer on the innermost side of the secondary thickening

Fig. 11 Pit pair between a narrow tracheary element $(T)$ and a parenchyma cell $(P)$. On the side of the tracheary element, a pseudotorus with plasmodesmata is present (arrows), while on the parenchyma side, a protective layer is formed $(P L)$

death and retain their protoplast longer than cells without pseudo-tori. Lachaud and Maurousset (1996) on the other hand, observed plasmodesmata crossing the cap-like thickening in S. torminalis completely, which would indicate that the formation of the secondary cap does not result in blocking the plasmodesmata. We agree that it is unlikely that pseudo-tori have this protective function since plasmodesmata piercing the electron dense cap were also seen in our samples.

It seems implausible that pseudo-tori have a function in water transport in a similar way as genuine tori (Bailey 1913; Pittermann et al. 2005). The morphology and position of pseudo-tori on the pit membrane is too variable to seal the pit aperture appropriately or to have another role in the hydraulic system. Moreover, pseudo-tori are not present on every pit membrane, which excludes a complete protection of the entire cell against air-seeding (Rabaey et 
al. 2006, in press; Jansen et al. 2007). Whether or not pseudo-tori play a role in cell differentiation cannot be concluded from this study. The close association between pseudo-tori and plasmodesmata suggests an interaction with functional and/or developmental aspects of plasmodesmata in vessels and fibre-tracheids.

\section{Notes on the protective layer}

A pseudo-torus can also be present on a pit membrane between a parenchyma cell and a tracheary element, but in that case only at the side of the tracheary element. At the parenchyma side, a discrete layer is formed. This layer is formed on pit membranes of so-called contact cells at the end of the secondary cell wall formation of the adjacent tracheary elements (Schaffer and Wisniewski 1989). Various functions have been suggested for this layer between the cell wall and the plasmalemma of the parenchyma cell. Schmid (1965) observed this 'protective layer' for the first time and suggested its protective role against the autolytic enzymes from the adjacent xylem element. Since this first report, similar structures have been observed in many other species. Several authors have provided evidence for its role in the formation of tyloses (Foster 1967; Murmanis 1975), freezing avoidance of the xylem (Wisniewski and Davis 1989), strengthening of the pit membrane, or facilitating intercellular transport (Van Bel and Van der Schoot 1988). All of these hypotheses, however, have been contradicted by other studies (Wooding and Northcote 1965; Chafe 1974; Wisniewski et al. 1987; Van Bel and Van der Schoot 1988; Wisniewski and Davis 1989; Barnett et al. 1993). Although the term 'protective layer' has commonly been used since Schmid (1965), it is misleading as the function of this conspicuous layer remains unclear and needs further investigation.

Acknowledgments We thank Annelies Pletsers (Royal Botanic Gardens, Kew) for assistance with the preparation of TEM sections. Prof. J. Rammeloo from the National Botanic Garden of Belgium is acknowledged for the permission to collect wood samples. We thank the SYNTHESYS program for financial support to DR (GB-TAF3115). Research at the Laboratory of Plant Systematics is supported by research grants from the K.U.Leuven (OT/05/35) and the Fund for Scientific Research-Flanders (Belgium) (G.0268.04 and G.0250.05). FL is a postdoctoral fellow of the Fund for Scientific ResearchFlanders (Belgium). Financial support to $\mathrm{SJ}$ was provided by a research grant from NERC (NE/E001122/1).

\section{References}

Bailey IW (1913) The preservation treatment of wood. II. The structure of the pit membranes in tracheids of conifers and their relation to the penetration of gases, liquids, and finely divided solids into green and seasoned wood. For Quart 11:12-20
Barnett JR (1981) Secondary xylem development. In: Barnett JR (ed) Xylem cell development. Castle House, Tunbridge Wells, p 307

Barnett JR (1982) Plasmodesmata and pit development in secondary xylem elements. Planta 155:251-260

Barnett JR (1987a) The development of fibre-tracheid pit membranes in Pyrus communis L. IAWA Bull 7:195-220

Barnett JR (1987b) Changes in the distribution of plasmodesmata in developing fibre-tracheid pit membranes of Sorbus aucuparia L. Ann Bot 59:269-279

Barnett JR, Harris JM (1975) Early stages of bordered pit formation in radiata pine. Wood Sci Technol 9:233-241

Barnett JR, Cooper P, Bonner LJ (1993) The protective layer as an extension of the apoplast. IAWA J 14:163-171

Butterfield BG, Meylan BA (1982) Cell wall hydrolysis in the tracheary elements of the secondary xylem. In: Baas P (ed) New perspectives in wood anatomy. Martinus Nijhoff/Dr W. Junk, The Hague, pp 71-84

Carr DJ (1976) Plasmodesmata in growth and development. In: Gunning BES, Robards AW (eds) Intercellular communication in plants: studies on plasmodesmata. Springer, Berlin, p 387

Catesson AM (1983) A cytochemical investigation of the lateral walls of Dianthus vessels. Differentiation and pit membrane formation. IAWA Bull 4:89-101

Chafe SC (1974) Cell wall formation and protective layer development in the xylem parenchyma of trembling aspen. Protoplasma 80:129-147

Chaffey NJ, Barnett JR, Barlow PW (1997) Cortical microtubule involvement in bordered pit formation in secondary xylem vessel elements of Aesculus hippocastanum L. (Hippocastanaceae): a correlative study using electron microscopy and indirect immunofluorescence microscopy. Protoplasma 197:64-75

Chaffey NJ, Barnett JR, Barlow PW (1999) A cytoskeletal basis for wood formation in angiosperm trees: the involvement of cortical microtubules. Planta 208:19-30

Chaffey NJ, Barlow PW, Barnett JR (2000) A cytoskeletal basis for wood formation in angiosperm trees: the involvement of microfilaments. Planta 210:890-896

Coleman CM, Prather BL, Valente MJ, Dute RR, Miller ME (2004) Torus lignification in hardwoods. IAWA Bull 25:435-448

Dute RR, Rushing AE (1988) Note on torus development in the wood of Osmanthus americanus (Oleaceae). IAWA Bull 9:41-51

Dute RR, Rushing AE (1990) Torus structure and development in the woods of Ulmus alata Michx., Celtis laevigata Willd., and Celtis occidentalis L. IAWA Bull 11:71-83

Dute RR, Rushing AE, Perry JW (1990) Torus structure and development in species of Daphne. IAWA Bull 11:113-123

Dute RR, Rushing AE, Freeman JD (1992) Survey of intervessel pit membrane structure in Daphne species. IAWA Bull $13: 113-123$

Dute RR, Martin AL, Jansen S (2004) Intervascular pit membranes with tori in wood of Planera aquatica J.F. Gmel. J Alab Acad Sci 75:7-21

Ehlers K, Kollmann R (2001) Primary and secondary plasmodesmata: structure, origin and functioning. Protoplasma 216:1-30

Esau K, Charvat I (1978) On vessel member differentiation in the bean (Phaseolus vulgaris L.). Ann Bot 42:665-677

Fengel D (1972) Structure and function of the membrane in softwoods bordered pits. Holzforschung 26:1-9

Foster RC (1967) Fine structure of tyloses in three species of the Myrtaceae. Austral J Bot 15:25-34

Funada R, Abe H, Furusawa O, Imaizumi H, Fukazawa K, Ohtani J (1997) The orientation and localization of cortical microtubules in differentiating conifer tracheids during cell expansion. Plant Cell Physiol 38:210-212

Funada R, Miura H, Shibagaki M, Furusawa O, Miura T, Fukatsu E, Kitin P (2001) Involvement of localized cortical microtubules in the formation of a modified structure of wood. J Plant Res 114:419-497 
Gunning BES, Hardham AR (1982) Microtubules. Ann Rev Plant Physiol 33:651-698

IAWA Committee (1989) IAWA list of microscopic features for hardwood identification. IAWA Bull 10:219-332

IAWA Committee (2004) IAWA list of microscopic features for softwood identification. IAWA J 25:1-70

Imamura Y, Harada H (1973) Electron microscopic study on the development of the bordered pit in coniferous tracheids. Wood Sci Technol 7:189-205

Jansen S, Choat B, Vinckier S, Lens F, Schols P, Smets E (2004) Intervascular pit membranes with a torus in the wood of Ulmus (Ulmaceae) and related genera. New Phytol 163:51-59

Jansen S, Sano Y, Choat B, Rabaey D, Lens F, Dute RR (2007) Pit membranes in tracheary elements of Rosaceae and related families: new records of tori and pseudo-tori. Amer J Bot 94:503-514

Juniper BE (1977) Some speculation on the possible roles of the plasmodesmata in the control of differentiation. J Theor Biol 66:583-592

Kerr T, Bailey IW (1939) The cambium and its derivative tissues. X. Structure, optical properties and chemical composition of so called middle lamella. J Arnold Arbor 15:327-349

Lachaud S, Maurousset L (1996) Occurrence of plasmodesmata between differentiating vessels and other xylem cells in Sorbus torminalis L. Crantz and their fate during xylem maturation. Protoplasma 191:220-226

Murmanis L (1975) Formation of tyloses in felled Quercus rubra L. Wood Sci Technol 9:3-14

O'Brien TP (1981) The primary xylem. In: Barnett JR (ed) Xylem cell development. Castle House, Tunbridge Wells, p 307

Oda Y, Mimura T, Hasezawa S (2005) Regulation of secondary cell wall development by cortical microtubules during tracheary element differentiation in Arabidopsis cell suspensions. Plant Physiol 137:1027-1036

Parameswaran N, Liese W (1969) On the formation and fine structure of septate wood fibers of Ribes sanguineum. Wood Sci Technol 3:271-286

Parameswaran N, Liese W (1973) Anomalous structures in bordered pits of fibre-tracheids of Ribes sanguineum. Wood and Fiber 5:76-79

Parameswaran N, Liese W (1981) Torus-like structures in interfibre pits of Pyrus and Prunus. IAWA Bull 2:89-93

Parameswaran N, Vidal Gomes A (1981) Fine structural aspects of helical thickenings and pits in vessels of Ligustrum lucidum Ait. (Oleaceae). IAWA Bull 2:179-185
Pittermann J, Sperry JS, Hacke UG, Wheeler JK, Sikkema EH (2005) Torus-margo pits help conifers compete with angiosperms. Science 310:1924

Rabaey D, Lens F, Smets E, Jansen S (2006) The micromorphology of pit membranes in tracheary elements of Ericales: new records of tori or pseudo-tori. Ann Bot 98:943-951

Rabaey D, Huysmans S, Lens F, Smets E, Jansen S (in press) Micromorphology and systematic distribution of pit membrane thickenings in Oleaceae: tori and pseudo-tori. IAWA J

Schaffer K, Wisniewski M (1989) Development of the amorphous layer (protective layer) in xylem parenchyma of $\mathrm{cv}$ Golden delicious apple, cv Loring peach, and willow. Amer J Bot 76: $1569-1582$

Schmid R (1965) The fine structure of pits in hardwoods. In: Côté WA (ed) Cellular ultrastructure of woody plants. Syracuse Univ. Press

Schmid R, Machado RD (1968) Pit membranes in hardwoods-fine structure and development. Protoplasma 66:185-204

Schols P, Dessein S, D'Hondt C, Huysmans S, Smets E (2002) Carnoy: a new digital measurement tool for palynology. Grana 41:124-126

Singh AP (1987) Fine structure of hydrolysed primary walls in tracheary elements of petiolar xylem in Eucalyptus delegatensis. Ann Bot 60:315-319

Srivastava LM, Singh AP (1972) Certain aspects of xylem differentiation in corn. Can J Bot 50:1795-1804

Uehara K, Hogetsu T (1993) Arrangement of cortical microtubules during formation of bordered pit in the tracheids of Taxus. Protoplasma 172:145-153

Van Bel AJE, Van der Schoot C (1988) Primary function of the protective layer in contact cells. Buffer against oscillations in hydrostatic pressure in the vessels. IAWA Bull 9:285-288

Wisniewski M, Davis G (1989) Evidence for the involvement of a specific cell wall layer in regulation of deep supercooling of xylem parenchyma. Plant Physiol 91:151-156

Wisniewski W, Ashworth E, Schaffer K (1987) Use of lanthanum to characterize cell wall permeability to deep supercooling and extracellular freezing in woody plants I. Intergeneric comparisons between Prunus, Pyrus, and Salix. Protoplasma 139:105-116

Wooding FBP, Northcote DH (1965) An anomalous wall thickening and its possible role in uptake of stem-fed tritiated glucose by Pinus picea. J Ultrastruct Res 12:463-472

Yang KC (1978) The fine structure of pits in yellow birch (Betula allaghaniensis Britton). IAWA Bull 4:71-77 